\title{
A Remote-operated System to Map Radiation Dose in the Fukushima Daiichi Primary Containment Vessel
}

\author{
M. Nancekievill, A. R. Jones, M. J. Joyce, Member, IEEE, B. Lennox, Senior Member IEEE, S. \\ Watson, Member, IEEE, J. Katakura, K. Okumura, S. Kamada, M. Katoh and K. Nishimura
}

\begin{abstract}
This paper describes the development of a submersible system based on a remote-operated vehicle coupled with radiation detectors to map the interior of the reactors at the Fukushima Daiichi nuclear power station. It has the aim oflocating fuel debris. The AVEXIS submersible vehicle used in this study has been designed as a low-cost, potentially disposable, inspection platform that is the smallest of its class and is capable of being deployed through a $150 \mathrm{~mm}$ diameter access pipe. To map the gamma-ray environment, a cerium bromide scintillator detector with a small form factor has been incorporated into the AVEXIS to identify radioactive isotopes via gamma-ray spectroscopy. This provides the combined system with the potential to map gamma-ray spectra and particle locations throughout submerged, contaminated facilities, such as Units 1, 2 and 3 of the Fukushima Daiichi nuclear power plant. The hypothesis of this research is to determine the sensitivity of the combined system in a submerged environment that replicates the combination of gamma radiation and water submersion but at lower dose rates.
\end{abstract}

Index Terms - Fukushima Daiichi, Gamma-ray detection, Radiation monitoring, Nuclear Decommissioning

\section{INTRODUCTION}

A fter the Fukushima nuclear power plant accident in 2011, many projects have been started with the aim of completing remote inspection and characterization of the inside of the stricken reactors [1]. Due to the evacuation of the surrounding area and flooding of the primary containment vessels (PCV), little is known of the current physical state of the reactor cores inside the PCVs of reactors 1, 2 and 3.

Although large portions of Fukushima Daiichi have been characterized using long-established remote methods, the current state of the environment at the bottom and beneath the submerged pedestal in the reactors is largely uncharacterized

${ }^{\mathrm{P}}$ aper submitted May $31^{\text {st }}, 2017$. This work was funded by the Engineering and Physical Sciences Research Council (EPSRC:EP/N017749/1) and MEXT (Japan). MJJ acknowledges the generous support of the Royal Society as a Wolfson Research Merit Award holder.

M. Nancekievill, B. Lennox and S. Watson were with the Department of Electrical and Electronic Engineering, University of Manchester, United Kingdom, UK e-mail: matthew.nancekievill@manchester.ac.uk
[2]. This leads to uncertainties in dose-rate levels and the extent of contaminated materials, and since dose-rate levels as high as $1 \mathrm{kGy} \mathrm{h}^{-1}$ are estimated, this could, if not better understood, hamper the progress of decommissioning the plant because of the potential for there to be prohibitive levels of total ionizing dose.

The AVEXIS vehicle was developed in the United Kingdom as part of a project to characterize the Sellafield Legacy ponds [3]. New designs and development cycles have now increased the robustness and adaptability of the vehicle to a multitude of environments.

A live feed camera is included to allow visual inspection of unknown environments with complete manual control of the system from the surface via a neutrally-buoyant, bespoke tether of diameter $<10 \mathrm{~mm}$.

The results of experimental testing of the remote-operated vehicle (ROV) with the integrated $\mathrm{CeBr}_{3}$ detector are described in this paper. Testing has been conducted in water tank facilities at both the University of Manchester and Lancaster to determine the capability of the detector to characterize the gamma spectrum from a known source positioned on the exterior of the tank.

Due to the modularity of the AVEXIS design, it is possible to replace the cerium bromide detector with other detectors. In particular the integration of a single crystal diamond chemical vapor deposition neutron detector with a ${ }^{6} \mathrm{Li}$ convertor foil able to map the thermal neutron flux and complementing the gamma-ray sensing functionality.

The remainder of the paper is structured as follows: Section II discusses improvements made to the ROV for suitable operational capacity to be deployed within the Fukushima Daiichi nuclear power plant. Section III introduces the integration of the cerium bromide detector and experimental results of the gamma spectrum analysis with a small ${ }^{137} \mathrm{Cs}$ source. Finally, section IV outlines the work to be completed in the future before the system can be deployed in the field.

A.R. Jones and M.J. Joyce are with the Department of Engineering, Lancaster University, United Kingdom.

J. Katakura is with the Nagoaka University of Technology, Japan

K. Okumura is with the Japan Atomic Energy Agency, Japan.

S. Kamada, M. Katoh, and K. Nishimura are with the National Maritime Research Institute, Japan. 


\section{AQUA VEHICLE EXPLORER FOR IN-SITU SENSING}

The Aqua Vehicle Explorer for In-situ Sensing (AVEXIS) is a submersible ROV developed by the University of Manchester and is shown in Figure 1. It was designed for characterization and monitoring of the Sellafield legacy ponds in hard-to-reach areas [3].

The ROV needs to be low-cost allowing easy disposal of the submersible, if required, due to the potential for there to be extensive contamination with radioactive materials. The net material cost for the device was approximately $£ 200$, which is consistent with the requirement that multiple ROVs could be manufactured and purchased at relatively easily if required.

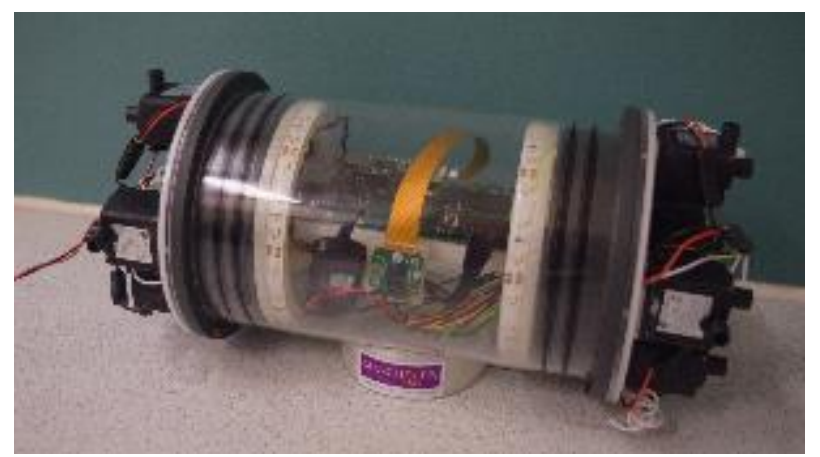

Figure 1 AVEXIS design.

\section{A. Overview of the AVEXIS}

AVEXIS is cylindrical in shape with an outer diameter of 150 $\mathrm{mm}$. It comprises 5 water pumps on each end-cap that provide movement forwards, backwards, up, down, left and right. Left and right motion occurs through the central axis; therefore the turning radius is half the length of the ROV. This makes the AVEXIS highly maneuverable, consistent with the requirement for characterization of hard-to-access areas.

The end caps are also designed with two O-rings for a watertight seal up to $100 \mathrm{~m}$ depth. They are also easily removable for adaptation and maintenance of the internal electronics, for replacement of the actuators or for modifications to the length of the vehicle if necessary.

A Raspberry $\mathrm{Pi}^{\mathrm{TM}}$ Zero is used for the main control of the ROV. This is connected to a Raspberry Pi ${ }^{\mathrm{TM}}$ Camera for a live video feed, displayed on the surface control station for visual inspection. An LED strip on each side of the ROV creates light to allow visual inspection in poorly lit water.

The neutrally-buoyant, custom tether consists of six 26 American Wire Gauge (awg) wires bundled into two sets of three (this will be referred to as a two-wire tether for simplicity). Both communication and power are afforded over this two-wire setup.

Powerline technology often found in homes has been adapted for use in the AVEXIS, to constitute a custom Power Over Ethernet (POE) system capable of $200 \mathrm{Mbps}$ transfer speeds. This is more than enough for High-definition (HD) live camera feed and instantaneous control of the ROV. This is very similar to the system used in OpenROV vehicles [4].

A local area network was created by attaching the local Ethernet port of a computer on the surface to a powerline adaptor. This communicates over the two-wire tether to a powerline adaptor inside the AVEXIS that is connected to a mini USB-to-Ethernet adaptor, connected to the Raspberry PiZero. With the Raspberry PiZero acting as a web-server, live video and control can be streamed to any web browser. A commercial gamepad such as a Sony PS4 or Microsoft Xbox controller can be used for intuitive control.

Power is supplied by placing a $48 \mathrm{~V}$ d.c. power supply in parallel to the two-wire tether. Communications were unaffected by any bias across the two wires. However, filters were placed on the input of each power supply to avoid interference from the $\mathrm{MHz}$ range frequency of Ethernet communication.

The development of a POE tether equated to a reduction in tether size to conventional ROVs as well as an increase in mechanical flexibility. This was especially important for a small ROV as it could be affected by a cumbersome tether reducing the working scope of characterization.

The main ROV board contains a DC-DC converter from 48 $\mathrm{V}$ d.c. to $12 \mathrm{Vd}$.c. for the pumps and $5 \mathrm{Vd}$.c. for the control electronics. The I/O electronics for the AVEXIS are designed around an ATMEGA 32U4, commonly found on an Arduino Leonardo.

\section{B. $\mathrm{CeBr}_{3}$ Gamma detector integration}

For the initial integration of the $\mathrm{CeBr}_{3}$ gamma detector in the $\mathrm{ROV}$, an additional tether was added that consisted of a RG178 coaxial cable. This was used to transmit the signal data from the detector to the surface for analysis. The weight and outer diameter of this additional tether was very small, which allowed it to be wrapped around the AVEXIS custom tether with no reduction in maneuverability or operational flexibility.

Power for the detector was delivered by the main ROV circuitry. This meant no additional power conversion circuit was required allowing quick integration of this detector. It also allows adaptability for future gamma detector/neutron detector integration.

\section{Other important specifications}

The AVEXIS was designed to carry a payload of approximately $1 \mathrm{~kg}$. This was to accommodate the neutron and gamma detectors and to provide contingency should other sensors be required. For example, a sonar could be attached for understanding the physical topology and dimensions of any debris in an environment where visual inspection is not possible.

Figure 2 shows the layout of one of the AVEXIS endcaps. It shows the tether access point, the coaxial connector for radiation detector signal propagation and the access point for the pump control wires. It also illustrates the position of a depth sensor capable of measuring depth to a tolerance of $\pm 2 \mathrm{~mm}$ [5].

The depth sensor allows for semi-autonomous control of the depth of the ROV and better control overall. Visual inspection does not allow for fine control of depth. Therefore, integration of a "depth lock" using this pressure sensor to autonomously maintain a constant depth was applied. This permitted greater security and safety in ROV movement.

Connectors for each individual water pump allow for quick and easy replacement if any pumps became damaged or faulty. This made the ROV modular in design and adaptable to changes in the required thrusters on each ROV. 
The use of commercial off-the-shelf components keeps the total manufacturing price low and caters for the prospect of the unit being lost during use.

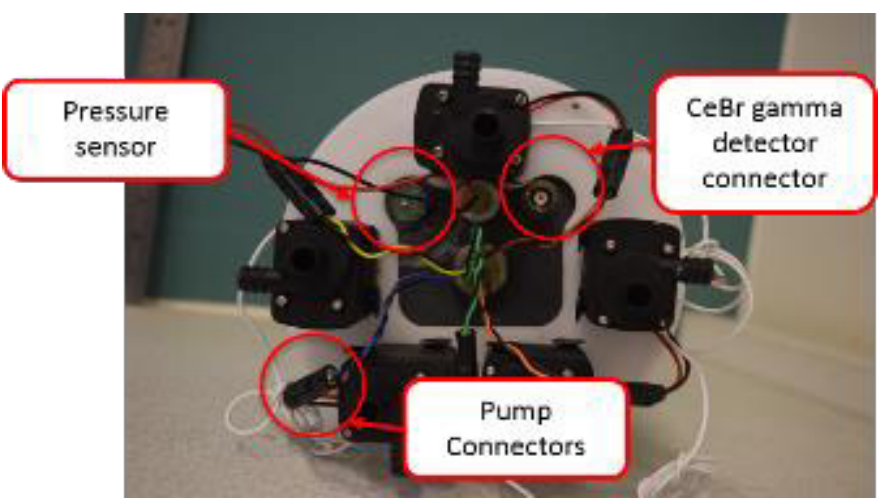

Figure 2 AVEXIS end-cap layout.

\section{CERIUM BROMIDE DETECTOR IMPLEMENTATION}

\section{A. Detector overview}

The detector used for these experiments is a $\mathrm{CeBr}_{3}$ inorganic scintillator that is sensitive to gamma radiation. $\mathrm{CeBr}_{3}$ was considered one of the best alternatives to replace the wellestablished $\mathrm{NaI}(\mathrm{Tl})$ because of its high detection efficiencies and improved energy resolution combined with resilience in high levels of radiation and room temperature operation.

The detector has a $10 \mathrm{~mm}$ diameter crystal coupled to photomultiplier with a small, integrated, high-voltage supply unit. The integrated HV supply allows the unit to be operated from a $5 \mathrm{~V}$ signal supplied via the ROV.

The detector unit was manufactured by Scionix [6], Netherlands, and the schematic diagram is shown in Figure 3. Each constituent part is labelled and explained in the schematic.

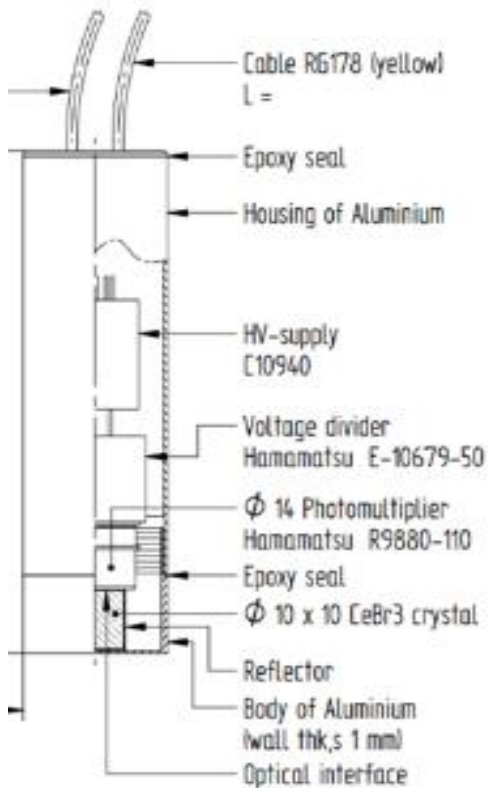

Figure $3 \mathrm{CeBr}_{3}$ inorganic scintillator detector, model VS-0087-50 supplied by Scionix, Netherlands [6].

For another layer of protection against an unlikely situation of potential leaks within the ROV whilst submerged, the detector was placed within an aluminium casing. The detector can be seen with and without the casing in Figure 4.

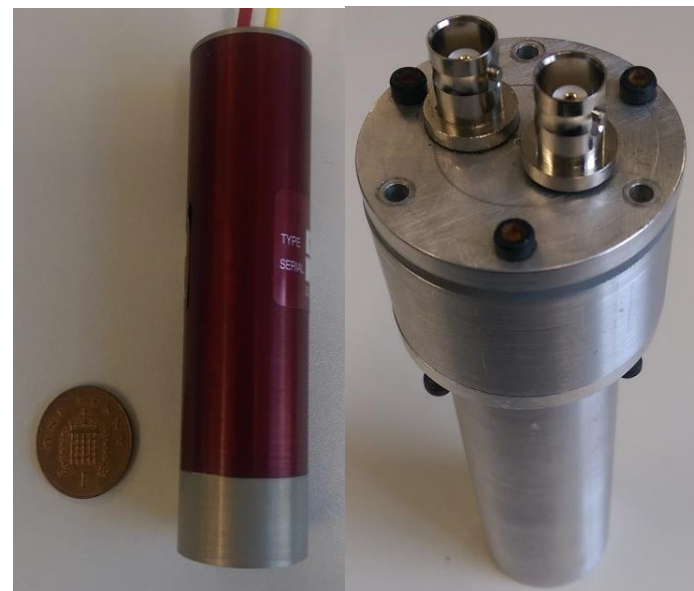

Figure 4 The $\mathrm{CeBr}_{3}$ inorganic scintillator detector without and with aluminium casing.

\section{B. Initial Characterisation}

Initial tests were carried out in the laboratory to investigate the impact the aluminium casing itself had on the response of the detector. These initial tests were carried out with both a 330 $\mathrm{kBq}{ }^{137} \mathrm{Cs}$, and a $50 \mathrm{kBq}{ }^{22} \mathrm{Na}$ source. The signal from the detector was passed through and processed by a single-channel, mixed-field analyser (MFA) manufactured by Hybrid Instruments Ltd. [7]. Comparative MCA plots from these tests can be seen in Figure 5 and Figure 6.

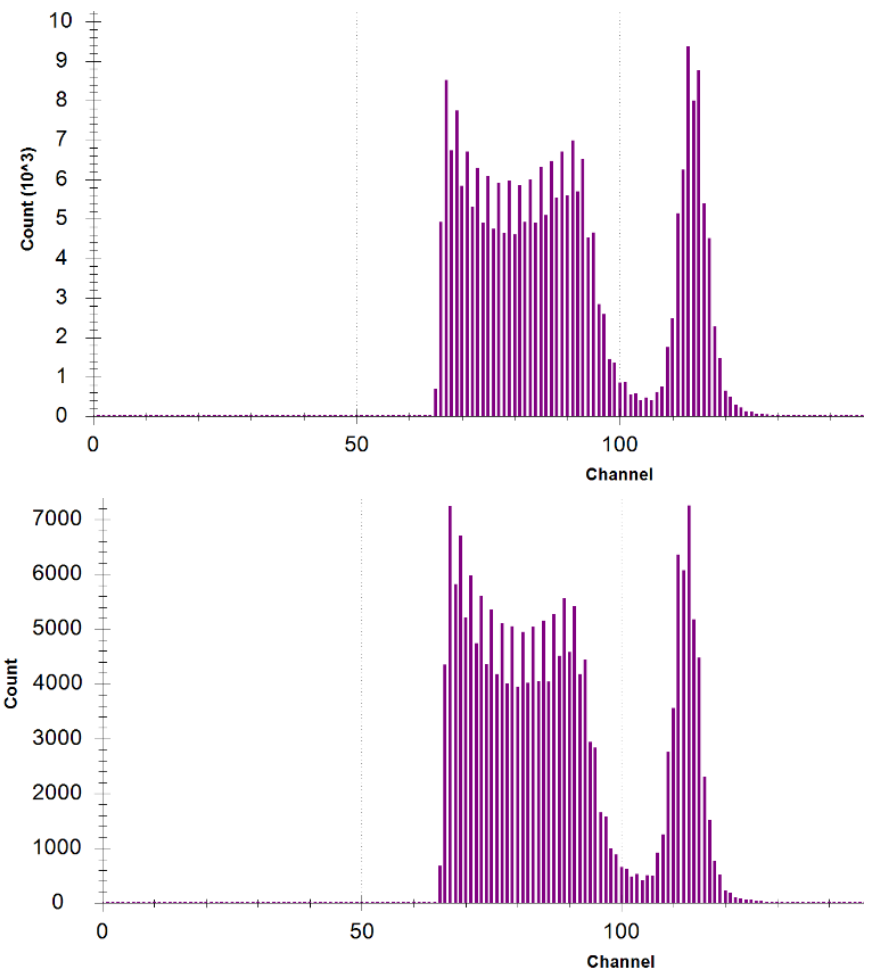

Figure $5 \mathrm{CeBr}_{3}$ detector exposed to ${ }^{137} \mathrm{Cs}$ source for 10 minutes. Top graph shows results without casing, bottom graph represents detector within casing. 

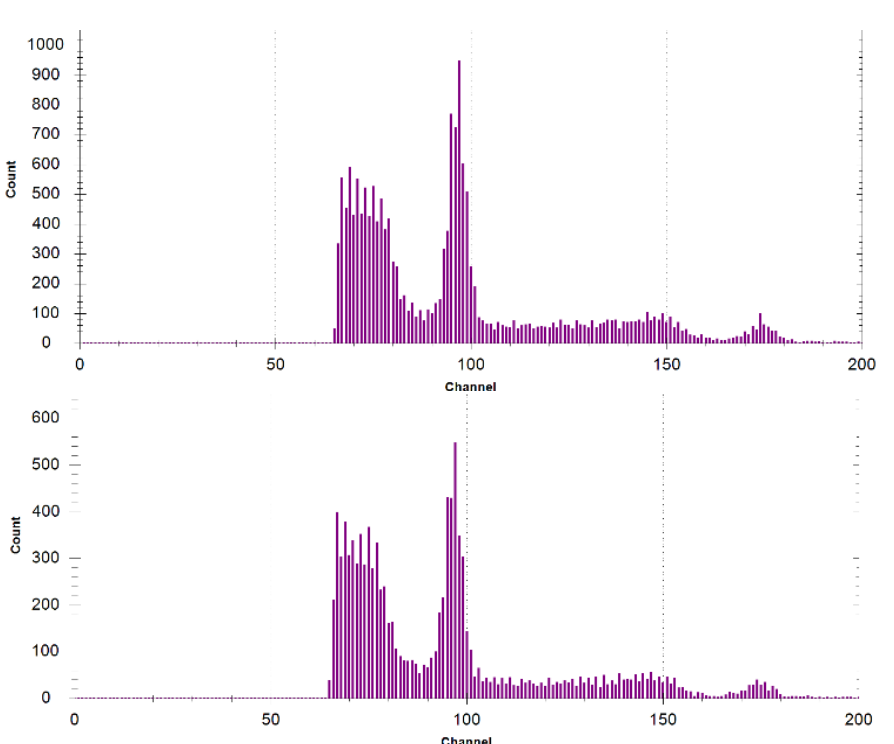

Figure $6 \mathrm{CeBr}_{3}$ detector exposed to ${ }^{22} \mathrm{Na}$ source for 10 minutes. Top graph shows results without casing, bottom graph represents detector within casing.

As can be seen from the MCA plots in Figure 5 and Figure 6 the spectral peaks are all consistent between the tests with and without the casing. However, the intensity of the peaks relative to each other does vary, as expected.

This intensity change can be observed by the throughput of counts observed by detector during this set time period with and without the casing. The results of this are shown in Table 1.

Table 1 Comparison of Number of Counts With and Without the Aluminium Casing

\begin{tabular}{lrlll}
\hline & \multicolumn{2}{c}{10 minutes } & \multicolumn{2}{c}{ Pulses per second } \\
\hline & No Casing & Casing & $\begin{array}{l}\text { No } \\
\text { Casing }\end{array}$ & Casing \\
\hline $\begin{array}{l}\text { Cs } \\
\text { source }\end{array}$ & $216291 \pm 465$ & $174552 \pm 418$ & $360 \pm 19$ & $291 \pm 17$ \\
\hline $\begin{array}{l}\text { Na } \\
\text { Source }\end{array}$ & $19705 \pm 140$ & $12485 \pm 112$ & $33 \pm 6$ & $21 \pm 5$ \\
\hline
\end{tabular}

As can be seen by the results in Table 1, the number of counts was significantly reduced when the casing was used. The difference in attenuation between the two sources is assumed to be associated with the different energy spectrums that are emitted. This is expected, with the lower-energy spectrum of $\mathrm{Na}$, dropping by a higher percentage than the Cs spectrum.

\section{Integrated with ROV experiments}

The next stage for the experiment was to integrate the detector within the ROV. A power supply from within the ROV was used to power the detector and the additional tether made from a RG178 coaxial cable sent back the readings from the detector. The experimental setup can be seen in Figure 7.

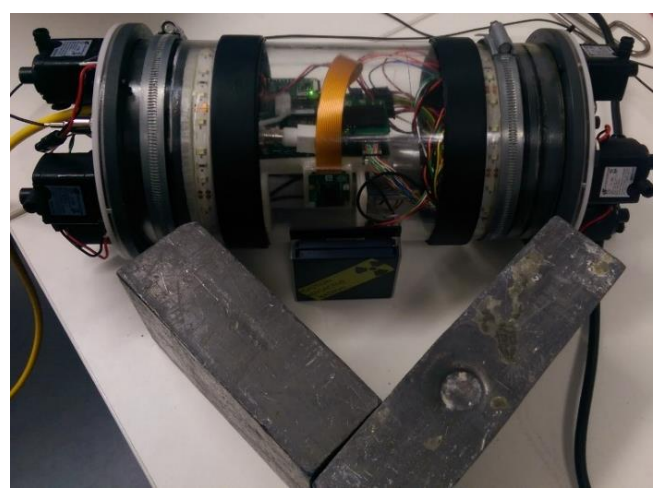

Figure 7 Benchtop test of gamma detector integrated within the ROV

The combined system was tested initially on the bench in the laboratory to ensure that the power supply from the ROV was sufficient for the detector to operate, as well as to confirm that the detector signal was transmitted successfully over a $50 \mathrm{~m}$ cable without degradation or noise overpowering the signal. The response of the detector to a 10-minute exposure to a small $330 \mathrm{kBq}{ }^{137} \mathrm{Cs}$ source located firstly inside and subsequently outside the ROV is shown in Figure 8.

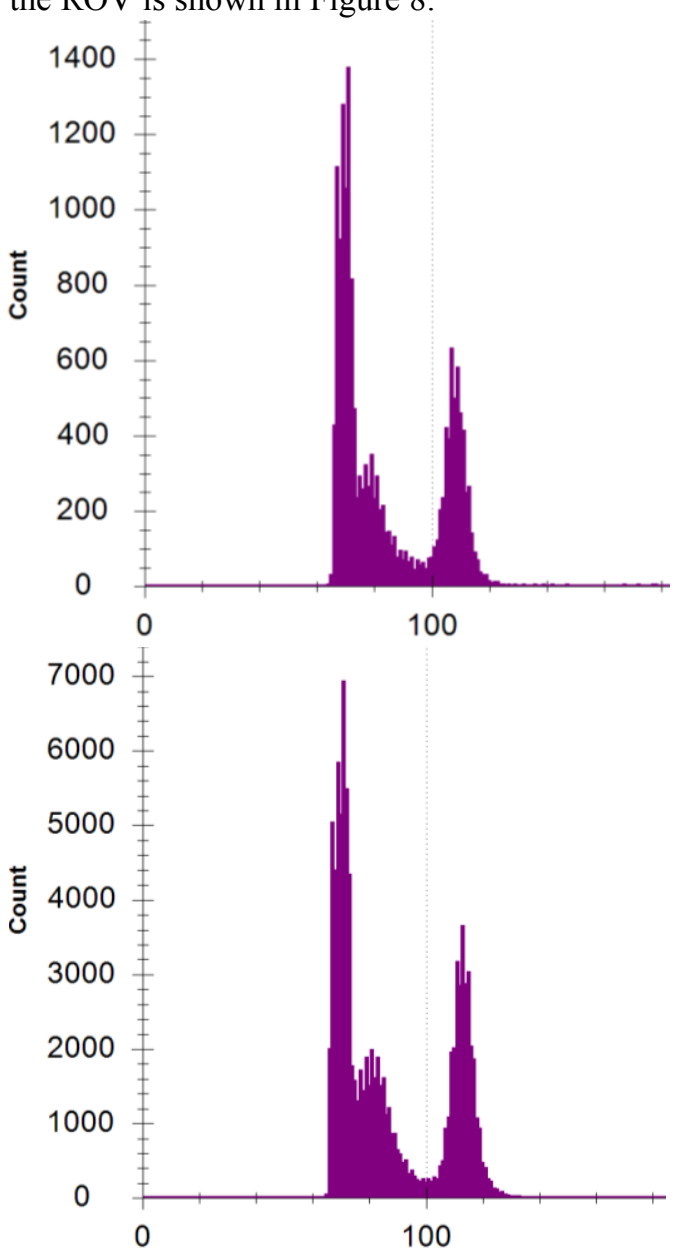

Figure $8 \mathrm{CeBr} 3$ detector exposed to a ${ }^{137} \mathrm{Cs}$ source for 10 minutes. Bottom, integrated within the ROV.

From Figure 8 it can be seen visually that the spectrum is less defined with a lower number of counts when the source was 
located outside the ROV. This is because of the additional distance and attenuation from the source when the detector was within the ROV unit. However, the results show that the detector is operational and able to function using the power supplied by the ROV.

The signal was transmitted via the $50 \mathrm{~m}$ tether and was sufficient to produce a spectrum. It is worthy to note that the signal was attenuated and settings on the data acquisition unit had to be adjusted to account for this in comparison to the previous test results shown in Figure 5 and Figure 6.

The ROV was then tested using a wave tank located at Lancaster University, UK. A ${ }^{137} \mathrm{Cs}$ source was placed on the side of the tank; the same source used in the previously described laboratory tests. A photograph of the experimental facilities is provided in Figure 9.

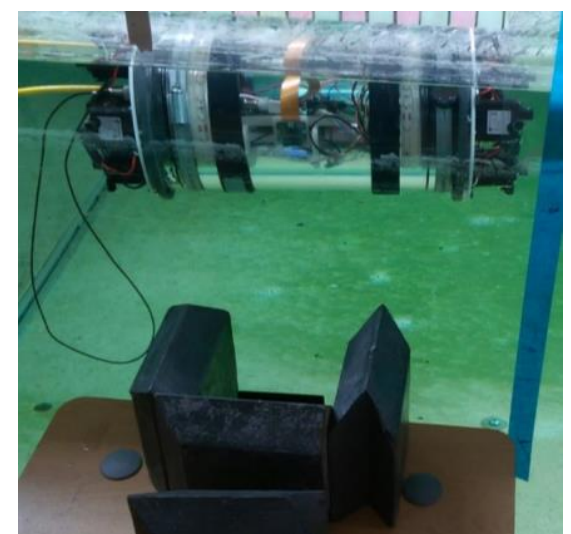

Figure 9 Submerged ROV gamma detector experiments. Cs source was placed up against the edge of the tank with the AVEXIS parallel to the source submerged.

Initial tests involved submerging the ROV with the integrated detector at two specific distances. The first was with the ROV submerged in the tank but as close to the wall as possible, whilst the second was with the ROV at an approximate distance of $0.5 \mathrm{~m}$ away from the side of the tank. Two 10minute exposures were again taken with the spectra provided for comparison purposes in Figure 10.

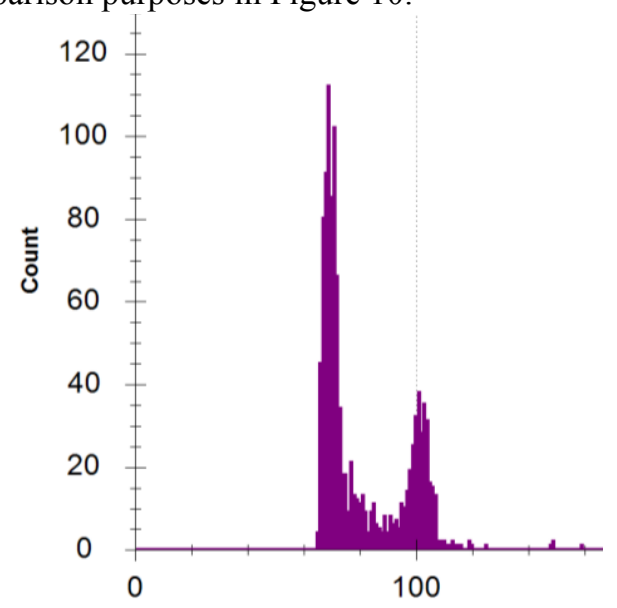

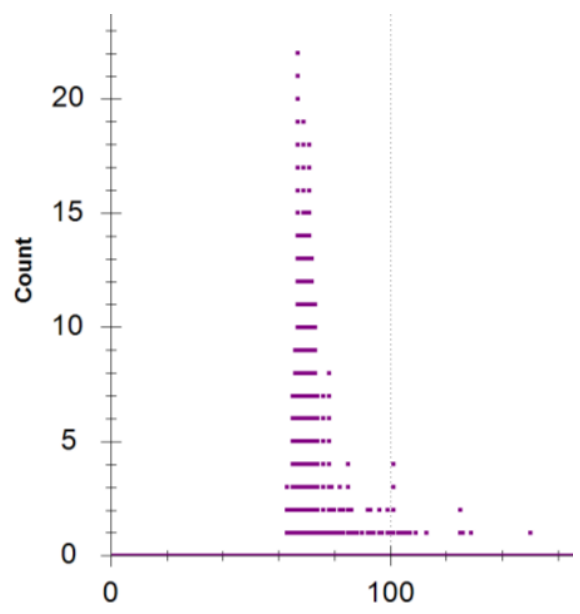

Figure 10 Detector exposed to a ${ }^{137}$ Cs source for 10 minutes whilst integrated within the ROV and submerged underwater. Top, with the ROV pressed against the side of the tank. Bottom, with the ROV approximately $0.5 \mathrm{~m}$ away.

These results show that due to the relatively small activity of the source, it was difficult to obtain a clear gamma-ray spectrum when the ROV was $0.5 \mathrm{~m}$ from the tank wall for a 10 -minute exposure. However, the outline of the spectrum is starting to become apparent and either a longer exposure or source of higher activity is anticipated to rectify this problem.

These tests demonstrate the detector is able to operate and provide data that can be used to identify a radioactive source whilst submerged and in very close proximity to a weak ${ }^{137} \mathrm{Cs}$ source. However, source identification from a greater distance in a submerged state was not possible.

In theory, whilst in a submerged environment, the $\mathrm{CeBr}_{3}$ gamma detector could take the number of counts over a set time period at different pre-determined positions could then be used to approximate source location; operating in total gamma mode. Using the approximate location of any source, the ROV could then be positioned so that the detector is exposed to the higher counts required to carry out the more detailed spectral analysis required for identification purposes. This two-stage analysis would aid in reducing the radiation damage to the ROV as proximity to the source is not reduced (and thus exposure to the radioactivity is not exacerbated) until necessary.

With a source of greater activity, a reduced time of exposure would be required to identify radioactive isotopes. In this research, an initial duration of 10 minutes was chosen to confirm the operational abilities of the system with a weak source. It is anticipated that isotopic identification will be possible at a much quicker rate, dependent on source activity and ROV distance. In the deployment, the level of activity is not expected to be a problem but, rather, the robustness of the system under high levels of exposure will need to be better understood.

\section{FUTURE WORK}

It is desirable to remove the extra tether access point for transmitting the detector measurements to reduce the likelihood of entanglement and to reduce weight of the tether. Therefore, 
methods to integrate the signal into the existing tether are being investigated to reduce the complexity of the tether and also to reduce the possibility of entanglement.

It is also anticipated that a neutron detector will be integrated into the AVEXIS to provide data about the fuel debris within the Fukushima Daiichi pedestal; this is distinct from the $137 \mathrm{Cs}$ as the latter is understood to be dispersed widely throughout the water covering the core, the suppression chamber and surrounding facility, thus not being uniquely associated with the debris. A scCVD diamond detector has been acquired and this can be seen in Figure 11.

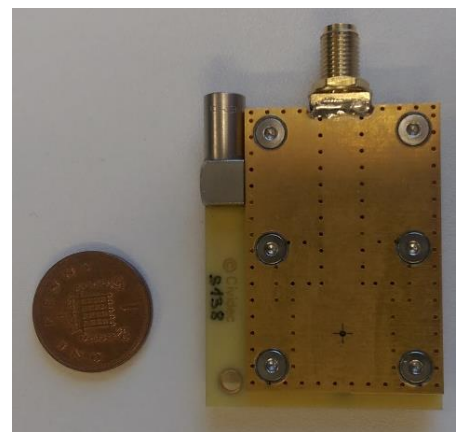

Figure 11 Neutron detector, scCVD diamond detector.

Before this device is installed it will be necessary to calibrate it using a high-energy source of alpha radioactivity. Testing will also need to be completed using a neutron source of significantly flux in order to be comparable with the Fukushima Daiichi environments.

A portable sonar will be added to the bottom of the ROV to aid in detection of debris, particularly suitable for in a murky environments in which the passage of light is impeded by suspended solids. The additional data provided by the sonar could be overlaid onto visual information gathered from the live video feed. A suitable sonar has been identified as the IMAGENEX 831L [8].

A number of options are being investigated to aid in the localisation of the ROV in an uncharted environment. Visual localisation can be achieved through a camera near the surface analysing the $x / y$ coordinates of the ROV, whilst the depth sensor adds $z$ data. Gamma spectrum analysis can then be overlaid onto this positional data.

Acoustic and RF options are being developed through the use of multiple transducers and receivers positioned along the length of the tether or boundaries of a tank. In water in which vision is impeded by suspended debris, the addition of sonar data will allow the build-up of a $3 \mathrm{D}$ point cloud, within which the ROV can localise itself.

\section{CONCLUSION}

Integration of a $\mathrm{CeBr}_{3}$ gamma detector into an underwater submersible capable of characterizing the inside of the Fukushima Daiichi nuclear power plant has been achieved.

Experimental validation has been achieved through benchtop and submerged testing. Further characterization of the capabilities of this device will be possible with a stronger gamma source and multiple submerged sources.

Improvements to both the submersible and gamma spectroscopy functions will be conducted with the aim of overlaying positional data of gamma sources in relation to the internal dimensions of the PCV. This will aid in the effort to decommission the nuclear power plant and lead to targeted removal of fuel debris, increasing safety to workers and efficiency of decommissioning.

\section{REFERENCES}

[1] IRID, "R \& D on Robots for the Decommissioning of Fukushima Daiichi NPS International Research Institute for Nuclear Decommissioning," Feb. 2016.

[2] IAEA, "Events and highlights on the progress related to recovery operations at Fukushima Daiichi Nuclear Power Station," 2017.

[3] A. Griffiths, A. Dikarev, P. Green, B. Lennox, X. Poteau, and S. Watson, "AVEXIS-Aqua Vehicle Explorer for In-Situ Sensing," IEEE Robotics and Automation Letters, vol. 3766, pp. 282-287, Jan-2016.

[4] OpenROV, "OpenROV 2.8 Kit," 2017. [Online]. Available: https://www.openrov.com/products/openrov28/. [Accessed: 14-Apr2017].

[5] TE connectivity, "MS5837-30BA Ultra Small Gel Filled Pressure Sensor," 2015.

[6] SCIONIX, "SCIONIX," 2017. [Online]. Available: http://scionix.nl/. [Accessed: 02-Jun-2017].

[7] H. I. Ltd., "Single Channel Mixed-Field Analyser," 2016. [Online]. Available: http://hybridinstruments.com/products/MFAX1.3.html. [Accessed: 15-Jun-2017].

[8] IMAGENEX, "IMAGENEX Model 831L Digital Pipe Profiling Sonar," 2011. 